\title{
Beyond the constraints of chemistry: Crystal structure discovery in particle simulation data
}

\author{
J. Dshemuchadse ${ }^{1,2}$, P.F. Damasceno ${ }^{1,3}$, C.L. Phillips ${ }^{4}$, S.C. Glotzer ${ }^{1}$, M. Engel $^{1,5}$ \\ ${ }^{1}$ University of Michigan, Ann Arbor, MI, USA, ${ }^{2}$ Cornell University, Ithaca, NY, USA, ${ }^{3}$ University of California, San Francisco, CA, \\ USA, ${ }^{4}$ Argonne National Laboratory, Argonne, IL, USA, ${ }^{5}$ Friedrich-Alexander University Erlangen-Nürnberg, Erlangen, Germany
}

michael.engel@fau.de

Do we know all conceivable crystal structures? This question appears naive at first, because crystallography is a mature field. But the list of reported inorganic crystal structures is not necessarily representative of all kinds of order that are possible on other scales. Atomic crystal structures are affected by the discreteness of the periodic table and the resulting constraints on chemical bonding. Molecular crystals, metal organic frameworks, nanoparticle superlattices, and other soft-matter assemblies are free from these chemical constraints and can exhibit entirely new types of crystallographic order distinct from those found with atoms. A universal list of all plausible crystal structures in systems of particles ranging from the angstrom to the micrometer scale would benefit the search for-and design of - new materials.

Here, we perform a data-driven simulation strategy to systematically crystallize one-component systems of particles interacting with isotropic multiwell pair potentials resembling Friedel oscillations and encoding and generalizing quantum mechanical interactions [1]. We investigate two tunable families of pairwise interaction potentials. Our simulations self-assemble a multitude of crystal structures ranging from basic lattices to complex networks. The goal is to discover crystal structures on the computer de novo, a strategy which has so far not been attempted on such a diverse set of systems. We perform a semi-automatic crystal structure analysis of simulation data. Our analysis reveals sixteen structures that have natural analogues spanning all coordination numbers found in inorganic chemistry. Fifteen more are hitherto unknown and occupy the space between covalent and metallic coordination environments. We describe the numerical search, the analysis technique, phase diagrams, and details of the known and previously unknown crystal structures. The discovered crystal structures constitute novel targets for self-assembly and expand our understanding of what a crystal structure can look like.

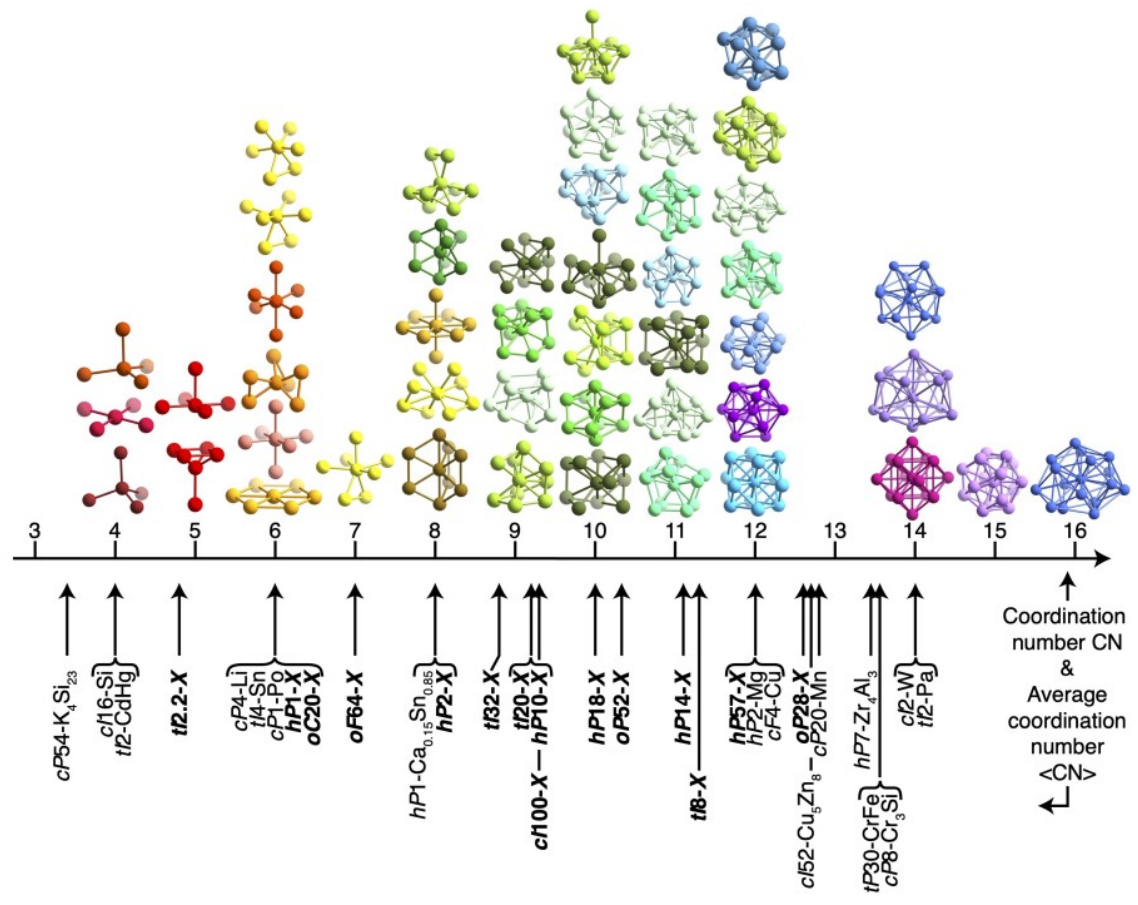

Figure 1. Coordination polyhedra in the 31 crystal structures self-assembled in our molecular dynamics simulations (displayed in colours representing their structures). Coordination polyhedra are grouped by coordination number $(\mathrm{CN})$ in the range $\mathrm{CN}=4-16$. Reported structures are listed by Pearson symbols below the $\mathrm{CN}$ axis and indicated by arrows pointing to their average coordination number $\langle\mathrm{CN}\rangle$ (the largest average coordination number observed is $\langle\mathrm{CN}\rangle=14$ ). Pearson symbols of previously unknown structures are labelled in bold font.

[1] Dshemuchadse, J., Damasceno, P.F., Phillips, C.L., Engel, M., Glotzer, S.C. (2021). Proc. Natl. Acad. Sci. U.S.A. 118, e2024034118.

Keywords: self-assembly; crystal structures; isotropic pair potentials 